\title{
DIFFUSION OF SPHERES IN A CONCENTRATED SUSPENSION II
}

\author{
C W J BEENAKKER and P MAZUR \\ Instituut-Lorentz, Rujksunversitent te Lelden, Nieuwsteeg 182311 SB Letden, The Netherlands
}

Recelved 17 January 1984

\begin{abstract}
We evaluate the wavevector dependent (short-time) diffusion coefficient $D(k)$ for spherical particles in suspension, by extending a previous study of selfdiffusion (which corresponds to the case of large $k$ ) Our analysis is valid up to high concentrations and fully takes into account the many-body hydrodynamic interactions between an arbitrary number of spheres, as well as the resummed contributions from a special class of correlatıons Results obtaned which agree well with avallable experimental data
\end{abstract}

\section{Introduction}

In a previous paper ${ }^{1}$ ) (hereafter referred to as I) we calculated the concentration dependence of the (short-time) selfdiffusion coefficient for spherical particles suspended in a fluid This quantity, denoted by $D_{s}$, is the large- $k$ limit of the wavevector dependent diffusion coefficient $D(k)$, which describes the initial decay of the dynamic structurefactor measured by inelastic light- or neutron-scattering ${ }^{23}$ ) In our analysis ${ }^{1}$ ) we resummed the contributions due to hydrodynamic interactions between an arbitrary number of spheres By including at most two-point correlations between the spheres, we obtained in paper I a reasonable agreement with experimental results ${ }^{4}$ ) for $D_{\mathrm{s}}$ for volume fractions $\phi \leqslant 03$ At higher concentrations the calculated values were too large, indicating the importance of higher order correlations

The extension to paper I presented here in twofold (I) we extend the formalism to diffusion at arbitrary values of the wavevector (ii) we resum to all orders the contributions from a special class of correlations

The (short-time) wavevector dependent diffusion coefficient $D(k)$ may be expressed in terms of the mobilities of the spheres ${ }^{2}$ ) To linear order in the density only two-sphere hydrodynamic interactions need to be considered and results for $D(k)$ to this order have been obtained by Russel and Glendinning ${ }^{5}$ ) and by Fijnaut ${ }^{6}$ ) In a suspension which is not dilute, however, it is essential to fully take into account the many-body hydrodynamic interactions between an arbitrary number of spheres The importance of non-additive hydrodynamic interactions was demonstrated theoretically in our calculation ${ }^{7}$ ) of the diffusion- 
coefficient to second order in the density, and experimentally by Pusey and van Megen's measurements ${ }^{4}$ ) of $D_{\text {s. }}$.

Using general expressions for many-sphere mobilities obtained by Mazur and van Saarloos $\left.^{8}\right)^{*}$, we shall give in section 2 a formula for the diffusion coefficient which is a convenient starting point for the calculation of $D(k)$ in a concentrated suspension. In the limit $k \rightarrow \infty$, this formula reduces to the expression for $D_{\text {s }}$ given in paper $\mathrm{I}$.

In sections 3, 4 and 5 we proceed to evaluate $D(k)$ through an expansion in correlationfunctions of higher and higher order. Such a "fluctuation expansion", in which the many-sphere hydrodynamic interactions are resummed algebraically, was employed in paper I also. However, here we resum-in addition - to all orders the contributions from a special class of correlations, the socalled "ring-selfcorrelations". Results $\dagger$ for the concentration and wavevector dependence of $D(k)$ are given in section 6 , and are compared to experimental data $^{4,11,12}$.

We conclude the paper in section 7 with an interpretation of our results in terms of an effective pair-mobility.

\section{An operator expression for $D(k)$}

As in paper I we study a system of $N$ spherical particles with radius $a$ and positionvectors $\boldsymbol{R}_{\imath}(i=1,2, \ldots, N)$, suspended in a liquid with viscosity $\eta$. While in our previous analysis we restricted ourselves to the self-diffusion coefficient $D_{\text {s }}$ of the suspended particles, we shall consider here the wavevector dependent diffusion coefficient $D(k)$, given by (see e.g. ref. 2)

$$
D(k)=k_{\mathrm{B}} T[N G(k)]^{-1} \sum_{i, j=1}^{N}\left\langle\hat{k} \cdot \mu_{i j} \cdot \hat{k} \mathrm{e}^{i k \cdot R_{i j}}\right\rangle .
$$

Here $\boldsymbol{k}$ is the wavevector with magnitude $k$ and direction $\hat{k} \equiv \boldsymbol{k} / k, G(k)$ is the static structure factor, $\boldsymbol{\mu}_{\imath}$ is a mobility tensor, $\boldsymbol{R}_{i j} \equiv \boldsymbol{R}_{j}-\boldsymbol{R}_{i}$, and $k_{\mathrm{B}}$ and $T$ denote Boltzmann's constant and the temperature, respectively. The angular brackets denote an average over the configurations of the spheres in a volume V.

The quantity defined in eq. (2.1) describes diffusion of the spheres on a timescale over which their positions are essentially constant ${ }^{2}$ ). It can be

\footnotetext{
* In this connection we mention that general expressions for many-sphere friction tensors were previously derived by Yoshızaki and Yamakawa9), by an analysis similar to that of ref 8 .

$\dagger$ These results have been published previously in ref 10
} 
measured by light-scattering, and is called in this context the "effective" diffusion coefficient ${ }^{3}$ ). The (short-time) selfdiffusion coefficient $D_{\mathrm{s}}$, studied in paper I, is given by

$$
D_{\mathrm{s}} \boldsymbol{\gamma}=k_{\mathrm{B}} T N^{-1} \sum_{i=1}^{N}\left\langle\boldsymbol{\mu}_{l l}\right\rangle .
$$

It is the large wavevector limit of $D(k)$

$$
D_{\mathrm{s}}=\lim _{k \rightarrow \infty} D(k)
$$

as can be understood by noting that

$$
\lim _{k \rightarrow \infty} G(k)=1
$$

and that in the limit $k \rightarrow \infty$ only the terms with $i=j$ contribute to the average in eq. (2.1). Note furthermore that, in an isotropic suspension, the average in eq. (2.2) is proportional to the unit tensor $\boldsymbol{t}$.

General expressions for the many-sphere mobility tensors $\mu_{i j}$ were derived by Mazur and van Saarloos ${ }^{8}$ ). It is convenient to write these results in the compact operator notation used in paper I. To this end we express the mobilities in terms of an operatorkernel $\boldsymbol{\mu}\left(\boldsymbol{r} \mid \boldsymbol{r}^{\prime}\right)$, by

$$
6 \pi \eta a \mu_{i j}=1 \delta_{i j}+\int \mathrm{d} \boldsymbol{r} \int \mathrm{d} \boldsymbol{r}^{\prime} \delta\left(\boldsymbol{r}-\boldsymbol{R}_{i}\right) \delta\left(\boldsymbol{r}^{\prime}-\boldsymbol{R}_{\jmath}\right) \boldsymbol{\mu}\left(\boldsymbol{r} \mid \boldsymbol{r}^{\prime}\right)
$$

We further define the microscopic number density $n(r)$ of the spheres

$$
n(\boldsymbol{r})=\sum_{i=1}^{N} \delta\left(\boldsymbol{r}-\boldsymbol{R}_{i}\right)
$$

Eq. (2.1) then takes the form

$$
G(k) D(k) / D_{0}=1+N^{-1} \int \mathrm{d} \boldsymbol{r} \mathrm{e}^{-i \boldsymbol{k} \cdot \boldsymbol{r}} \int \mathrm{d} \boldsymbol{r}^{\prime} \mathrm{e}^{\mathbf{k} \cdot \boldsymbol{r}^{\prime}}\left\langle\hat{k} \cdot n(\boldsymbol{r}) \boldsymbol{\mu}\left(\boldsymbol{r} \mid \boldsymbol{r}^{\prime}\right) n\left(\boldsymbol{r}^{\prime}\right) \cdot \hat{k}\right\rangle,
$$

or, defining the operators $\boldsymbol{\mu}$ with kernel $\boldsymbol{\mu}\left(\boldsymbol{r} \mid \boldsymbol{r}^{\prime}\right)$ and $n$ with kernel $n(\boldsymbol{r}) \delta\left(\boldsymbol{r}^{\prime}-\boldsymbol{r}\right)$,

$$
G(k) D(k) / D_{0}=1+N^{-1}\langle\hat{k} \cdot\{n \mu n\}(k \mid k) \cdot \hat{k}\rangle .
$$

In this last equation we have defined the Fourier transform of an operator- 
kernel $O\left(\boldsymbol{r} \mid \boldsymbol{r}^{\prime}\right)=\{n \boldsymbol{\mu} n\}\left(\boldsymbol{r} \mid \boldsymbol{r}^{\prime}\right)$ as

$$
O\left(k \mid k^{\prime}\right) \equiv \int \mathrm{d} r \mathrm{e}^{i k r} \int \mathrm{d} \boldsymbol{r}^{\prime} \mathrm{e}^{i k r} O\left(\boldsymbol{r} \mid \boldsymbol{r}^{\prime}\right)
$$

The Stokes-Einstein diffusion coefficient is denoted by

$$
D_{0}=k_{\mathrm{B}} T(6 \pi \eta a)^{1}
$$

Adopting the notation of paper I we may wite (see below) for the operator $\mu$

$$
\boldsymbol{\mu}=P \mathscr{A}\left(1-n Q \mathscr{B}^{-1} \mathscr{A}\right)^{-1} P,
$$

and we thus finally obtain for the diffusion coefficient the expression

$$
G(k) D(k) / D_{0}=1+N^{-1}\left\langle\hat{k} \cdot\left\{P n \mathscr{A}\left(1-n Q \mathscr{B}^{-1} \mathscr{A}\right)^{-1} n P\right\}(k \mid k) \cdot \hat{k}\right\rangle
$$

We shall now show that expression (211) for the operator $\boldsymbol{\mu}$ is indeed equivalent to the general expressions for the mobility tensors given in ref 8 We shall first briefly recall the meaning of the symbols $\mathscr{A}, \mathscr{B}{ }^{1}, P$ and $Q$ used in eqs $(211)$ and $(212)$, cf section 3 in paper I The matrices $\mathscr{A}$ and $\mathscr{B}^{-1}$ have elements

$$
\{\mathscr{A}\}_{n m}=\tilde{\boldsymbol{A}}^{(n m)}, \quad\left\{\mathscr{B}^{-1}\right\}_{n m}=\delta_{n m} \boldsymbol{B}^{(m m) '},
$$

which are tensors of rank $n+m(n, m=1,2,3, \quad)$, the projection matrices $P$ and $Q=1-P$ have elements

$$
\{P\}_{n m}=\delta_{n 1} \delta_{m 1}, \quad\{Q\}_{n m}=\delta_{n m}-\delta_{n 1} \delta_{m 1}
$$

The tensor $\tilde{\boldsymbol{A}}^{(n m)}$ is a convolution operator with kernel

$$
\tilde{\boldsymbol{A}}^{(n m)}\left(\boldsymbol{r} \mid \boldsymbol{r}^{\prime}\right)=\tilde{\boldsymbol{A}}^{(n m)}\left(\boldsymbol{r}^{\prime}-\boldsymbol{r}\right)= \begin{cases}0 & \text { if } \boldsymbol{r}=\boldsymbol{r}^{\prime}, \\ \boldsymbol{A}^{(n m)}\left(\boldsymbol{r}^{\prime}-\boldsymbol{r}\right) & \text { if } \boldsymbol{r} \neq \boldsymbol{r}^{\prime}\end{cases}
$$

Convenient expressions for the constant tensor $\boldsymbol{B}^{(m m)^{-1}}$ and for the Fourier transform of $\boldsymbol{A}^{(n m)}(\boldsymbol{r})$,

$$
\boldsymbol{A}^{(n m)}(\boldsymbol{k})=\int \mathrm{d} \boldsymbol{r} \mathrm{e}^{i k r} \boldsymbol{A}^{(n m)}(\boldsymbol{r})
$$

are given in eqs (I-2 15) and (I-2 22)*

* The tensors $\boldsymbol{A}_{l j}^{(n m)}=\tilde{\boldsymbol{A}}^{(n m)}\left(\boldsymbol{R}_{t j}\right)$ and $\boldsymbol{B}^{(m m) t}$ were introduced by Mazur and van Saarloos $\left.{ }^{8}\right)$ These so-called "connectors" correspond to the "hydrodynamic interaction tensors" used previously by Yoshızakı and Yamakawa"), in order to discuss many-sphere friction tensors 
With these notations we may write e.g.,

$$
\begin{aligned}
\left\{P \mathscr{A} n Q \mathscr{B}^{-1} \mathscr{A} P\right\}\left(\boldsymbol{R}_{\imath} \mid \boldsymbol{R}_{\jmath}\right)= & \sum_{m=2}^{\infty} \int \mathrm{d} \boldsymbol{r}^{\prime \prime} \tilde{\boldsymbol{A}}^{(1, m)}\left(\boldsymbol{r}^{\prime \prime}-\boldsymbol{R}_{\imath}\right) n\left(\boldsymbol{r}^{\prime \prime}\right) \odot \boldsymbol{B}^{(m, m)^{-1}} \\
& \odot \tilde{\boldsymbol{A}}^{(m, 1)}\left(\boldsymbol{R}_{\jmath}-\boldsymbol{r}^{\prime \prime}\right) \\
= & \sum_{m=2}^{\infty} \sum_{\substack{k=1 \\
k \neq l, j}}^{N} \boldsymbol{A}_{l k}^{(1, m)} \odot \boldsymbol{B}^{(m, m)^{-1}} \odot \boldsymbol{A}_{k j}^{(m, 1)}
\end{aligned}
$$

where $\boldsymbol{A}_{l j}^{(n, m)} \equiv \tilde{\boldsymbol{A}}^{(n, m)}\left(\boldsymbol{R}_{j}-\boldsymbol{R}_{\imath}\right)$ and the $\operatorname{dot} \odot$ prescribes an $m$-fold contraction. By expanding the inverse operator in eq. (2.11) in powers of $n$, we obtain (after substitution into eq. (2.5)) the expression for $\boldsymbol{\mu}_{i j}$ derived in ref. 8 and given in eq. (I-2.2).

The expression (2.12) for the diffusion coefficient $D(k)$ is exact and fully contains the many-body hydrodynamic interactions between the $N$ spheres. It is the required extension of the formula for the selfdiffusion coefficient $D_{\text {s }}$ given in paper I, eq. (I-3.16). As we have shown there-and will see again in the next section - such formal operator expressions are very useful in a study of concentrated suspensions.

\section{Renormalization of the connectors}

Let $\gamma_{0}^{(m, m)}(m=1,2,3, \ldots)$ be an arbitrary constant tensor of rank $2 m$. We denote by $\gamma_{0}$ the diagonal matrix with elements

$$
\left\{\gamma_{0}\right\}_{n, m}=\delta_{n m} \gamma_{0}^{(m, m)}
$$

A matrix of renormalized connectors $\mathscr{A}_{\gamma_{0}}$ is defined - for each $\gamma_{0}$ - as

$$
\mathscr{A}_{\gamma_{0}} \equiv \mathscr{A}\left(1-\gamma_{0} Q \mathscr{B}^{-1} \mathscr{A}\right)^{-1}
$$

The $n, m$ element of the matrix $\mathscr{A}_{\gamma_{0}}$ is a renormalized connector $\boldsymbol{A}_{\gamma_{0}}^{(n, m)}$, which in turn is a convolution operator with kernel $\boldsymbol{A}_{\gamma_{0}}^{(n, m)}(\boldsymbol{r})$.

We now choose $\gamma_{0}^{(m, m)}$ to be a function of the average numberdensity of the spheres $n_{0}=N / V$,

$$
\begin{aligned}
& \boldsymbol{\gamma}_{0}^{(1,1)}=n_{0} \boldsymbol{1} \\
& \boldsymbol{\gamma}_{0}^{(m, m)}-\boldsymbol{\gamma}_{0}^{(m, m)} \odot \boldsymbol{B}^{(m, m)^{-1}} \odot \boldsymbol{A}_{\gamma_{0}}^{(m, m)}(\boldsymbol{r}=0)=n_{0} \boldsymbol{1}^{(m, m)}, \quad m \geqslant 2 .
\end{aligned}
$$


The tensor $\boldsymbol{1}^{(m, m)}$ used in this equation is a generalized unit tensor of rank $2 m$,

$$
\boldsymbol{f}^{(2,2)}=\boldsymbol{\Delta}^{(2,2)}, \quad \boldsymbol{1}^{(m, m)}=\boldsymbol{\Delta}^{(m-1, i d, m-1)} \quad(m \geqslant 3),
$$

where the $\boldsymbol{\Delta}$-tensors are defined in eqs. (I-2.9) and (I-2.19). The renormalized "density" $\gamma(\boldsymbol{r})$, with average $\gamma_{0}$, is given by

$$
\gamma(\boldsymbol{r}) \equiv \gamma_{0} n_{0}^{-1} n(\boldsymbol{r})
$$

the corresponding diagonal operator $\gamma$ has kernel $\gamma(\boldsymbol{r}) \delta\left(\boldsymbol{r}^{\prime}-\boldsymbol{r}\right)$. The renormalized density and connectors defined above will be explicitly evaluated in section 4.

In paper I we defined renormalized connectors $\mathscr{A}_{n_{0}}$ according to eq. (3.2), with $\gamma_{0}$ replaced by $n_{0}$, and used the identity

$$
\mathscr{A}\left(1-n Q \mathscr{B}^{-1} \mathscr{A}\right)^{-1} n=\mathscr{A}_{n_{0}}\left(1-\delta n Q \mathscr{B}^{-1} \mathscr{A}_{n_{0}}\right)^{-1} n,
$$

where $\delta n \equiv n-n_{0}$ denotes the density fluctuations. If one substitutes this identity into eq. (2.12) and expands the operator between braces in this equation in powers of $\delta n$, one obtains an expansion for $D(k)$ in correlationfunctions of higher and higher order (a so-called fluctuation expansion). For the case of selfdiffusion, this expansion was evaluated to second order in paper I. The renormalized connectors $\mathscr{A}_{n_{0}}$ account for a full resummation of the many-body hydrodynamic interactions in the absence of correlations, and in this way for the fact that (in some averaged sense) spheres interact hydrodynamically via a suspension with density $n_{0}$, rather than through the pure fluid. As we shall shortly see, the renormalization of the density, defined in eq. (3.3), will moreover account for a partial resummation of correlations.

The following identity will prove very useful in our analysis

$$
\mathscr{A}\left(1-n Q \mathscr{B}^{-1} \mathscr{A}\right)^{-1} n=\mathscr{A}_{\gamma_{0}}\left(1-\delta \gamma Q \mathscr{B}^{-1} \mathscr{A}_{\gamma_{0}}\right)^{-1} \gamma
$$

This formula differs from the previous one (eq. (3.6)) in that it contains the renormalized density $\gamma$, density fluctuations $\delta \gamma \equiv \gamma-\gamma_{0}$ and cut-out connectors ${\stackrel{\circ}{\boldsymbol{A}_{0}}(n, m)}_{\gamma_{0}}^{(n)}=\left\{\mathscr{A}_{\gamma_{0}}\right\}_{n, m}$ with kernels

$$
\AA_{\gamma_{0}}^{(n, m)}\left(\boldsymbol{r} \mid \boldsymbol{r}^{\prime}\right)=\AA_{\gamma_{0}}^{(n, m)}\left(\boldsymbol{r}^{\prime}-\boldsymbol{r}\right)= \begin{cases}0 & \text { if } \boldsymbol{r}=\boldsymbol{r}^{\prime} \text { and } n=m, \\ \boldsymbol{A}_{\gamma_{0}}^{(n, m)}\left(\boldsymbol{r}^{\prime}-\boldsymbol{r}\right) & \text { if } \boldsymbol{r} \neq \boldsymbol{r}^{\prime} \text { or } n \neq m .\end{cases}
$$

A proof of eq. (3.7) is given in the appendix. Substituting this identity into 
expression (2 12) for $D(k)$ one finds

$$
G(k) D(k) / D_{0}=1+N{ }^{1}\left\langle\hat{k} \cdot\left\{P n \mathscr{A}_{\gamma_{0}}\left(1-\delta \gamma Q \mathscr{B}^{-1} \mathscr{A}_{\gamma_{0}}\right)^{1} n P\right\}(\boldsymbol{k} \mid \boldsymbol{k}) \cdot \hat{k}\right\rangle,
$$

where use has been made of the fact that $\gamma P=n P$, in view of definitions (3 3) and (3 5)

If one expands the operator between braces in eq (39) in powers of $\delta \gamma$ one obtains again an expansion for $D(k)$ in density correlationfunctions, since $\delta \gamma=\gamma_{0} n_{0}^{-1} \delta n$ (cf eq (35)) is linear in the density fluctuations $\delta n$ The $\delta \gamma$ expansion differs however from the $\delta n$-expansion considered in paper I, in that the contributions from a special class of correlations (which we call ringselfcorrelations) are in the former expansion included in the lowest order term Indeed each term in the $\delta \gamma$-expansion may be obtained by partial resummation of the $\delta n$-expansion

The difference between these two expansions of the diffusion coefficient may be understood as follows An $s$-point correlation $\left\langle\delta n\left(\boldsymbol{r}_{1}\right) \delta n\left(\boldsymbol{r}_{2}\right) \quad \delta n\left(\boldsymbol{r}_{s}\right)\right\rangle$ contains many terms which are proportional to deltafunctions $\delta\left(\boldsymbol{r}_{k}-\boldsymbol{r}_{l}\right)(k, l=$ $1,2, \quad, s, k \neq l)$ For $s=2$ one has e $\mathrm{g}$

$$
\left\langle\delta n\left(\boldsymbol{r}_{1}\right) \delta n\left(\boldsymbol{r}_{2}\right)\right\rangle=n_{0} \delta\left(\boldsymbol{r}_{2}-\boldsymbol{r}_{1}\right)+n_{0}^{2}\left[g\left(\left|\boldsymbol{r}_{2}-\boldsymbol{r}_{1 \mid}\right|\right)-1\right],
$$

where the deltafunction term represents the selfcorrelation and $g(r)$ is the pair distributionfunction As a consequence of selfcorrelations, an expression of the form $\left\langle\left(\delta n \mathscr{A}_{n_{0}}\right)^{s}\right\rangle$ contains a class of contributions with factors $\boldsymbol{A}_{n_{0}}^{(m k)}(\boldsymbol{r}=0)$ $(m, k=1,2,3$, ) Referring to a diagrammatic representation, this factor is called a ring-selfcorrelation We remark that a contribution from these ringselfcorrelations is most important when the upper indices $m$ and $k$ of the factor $\boldsymbol{A}_{n_{0}}^{(m k)}(\boldsymbol{r}=0)$ are equal* In this case we speak of diagonal ring-selfcorrelations

Similarly, an $s$ th order correlation between renormalized density fluctuations $\left\langle\left(\delta \gamma \mathscr{A}_{\gamma_{0}}\right)^{s}\right\rangle$ would contain terms with factors $\AA_{\gamma_{0}}^{(m k)}(\boldsymbol{r}=0)$ However, in view of definition (38) of the cut-out connectorfield, these terms are zero, unless $m \neq k$ For this reason the various terms in the $\delta \gamma$-expansion do not contain diagonal ring-selfcorrelations The contributions of these have been resummed algebraically by the renormalization of the density through eq (3 3)

To conclude this section we give the expression for the selfdiffusion coefficient $D_{\mathrm{s}}$, which follows from eq (I-3 16), with the use of identity (37),

$$
1 D_{\mathrm{s}} / D_{0}=1+n_{0}^{-1}\left\langle\left\{P \mathscr{A}_{\gamma_{0}}\left(1-\delta \gamma Q \mathscr{B}^{-1} \mathscr{A}_{\gamma_{0}}\right)^{-1} n P\right\}(\boldsymbol{r} \mid \boldsymbol{r})\right\rangle
$$

\footnotetext{
* For example, the contribution (of second order in $\delta n$ ) to the selfdiffusion coefficient from the term with the factor $\boldsymbol{A}_{n_{0}}^{(2)}(r=0)$ is $-0084 D_{0}$, at the highest density considered in paper I (cf table II in paper I) At the same density, the term with the factor $\boldsymbol{A}_{n_{0}}^{(13)}(\boldsymbol{r}=0)$ contributes only $-0002 D_{0}$
} 
Note that, due to translational invariance, the r.h.s. of this equation is independent of $\boldsymbol{r}$. We recall that, as indicated in section $2, D_{\mathrm{s}}$ is also the large wavevector limit of $D(k)$, given by eq. (3.9). One must realize, however, that if one first expands the r.h.s. of eq. (3.9) in correlationfunctions of $\delta n$ of higher and higher order, this seriesexpansion is not equal term by term, in the limit $k \rightarrow \infty$, to the corresponding seriesexpansion of eq. (3.11). We shall return to this point in section 5 .

\section{Evaluation of the renormalized connectors}

In order to solve eq. (3.3) for $\gamma_{0}$ we shall make the following "Ansatz"

$$
\gamma_{0}^{(m, m)}=\gamma_{0}^{(m)} \boldsymbol{1}^{(m, m)}, \quad m \geqslant 2
$$

where $\gamma_{0}^{(m)}$ is a scalar function of the density $n_{0}$. As we shall see, this is indeed the form of the solution. The generalized unit tensor $\boldsymbol{1}^{(m, m)}$ was defined in eq. (3.4) and has the property that

$$
\boldsymbol{1}^{(m, m)} \odot \boldsymbol{B}^{(m, m)^{-1}}=\boldsymbol{B}^{(m, m)^{-1}}
$$

The evaluation of the renormalized connectorfield $\boldsymbol{A}_{\gamma_{0}}^{(n, m)}(\boldsymbol{r})$, defined in section 3 , then proceeds entirely as the evaluation of $\boldsymbol{A}_{n_{0}}^{(n, m)}(\boldsymbol{r})$ in paper I, section 6 , and gives

$$
\boldsymbol{A}_{\gamma_{0}}^{(n, m)}(\boldsymbol{r})=\tilde{\boldsymbol{A}}^{(n, m)}(\boldsymbol{r})-(2 \pi)^{-3} \int \mathrm{d} \boldsymbol{k} \mathrm{e}^{-i k \cdot r} \boldsymbol{A}^{(n, m)}(\boldsymbol{k}) \phi S_{\gamma_{0}}(a k)\left[1+\phi S_{\gamma_{0}}(a k)\right]^{-1}
$$

Here $\phi=(4 / 3) \pi a^{3} n_{0}$ is the volumefraction of the spheres and the function $S_{\gamma_{0}}(a k)$ is given as an infinite sum of Bessel functions

$$
S_{\gamma_{0}}(a k)=\sum_{p=2}^{\infty} \frac{9}{2} \varepsilon_{p} \gamma_{0}^{(p)} n_{0}^{-1}(2 p-1)^{2} \frac{\pi}{2}(a k)^{-3} J_{p-1 / 2}^{2}(a k)
$$

We have defined $\varepsilon_{2}=5 / 9, \varepsilon_{p}=1(p \geqslant 3)$. The case considered in paper I corresponds to $\gamma_{0}^{(p)} \equiv n_{0}$ for all $p$. The series in eq. (4.4) can then be summed analytically and gives the function $S(a k)$ defined in eq. (I-6.6).

To calculate the density dependence of $\gamma_{0}$, given by eq. (3.3), we need the 
result

$$
\begin{aligned}
\boldsymbol{B}^{(m, m)^{-1}} \odot \boldsymbol{A}_{\gamma_{0}}^{(m, m)}(\boldsymbol{r}=0)= & \boldsymbol{1}^{(m, m)}(2 m-1) \int_{0}^{\infty} \mathrm{d} k k^{-1} J_{m-1 / 2}^{2}(k) \phi S_{\gamma_{0}}(k) \\
& \times\left[1+\phi S_{\gamma_{0}}(k)\right]^{-1}, \quad m \geqslant 2,
\end{aligned}
$$

and for later use also

$$
\begin{aligned}
& \boldsymbol{A}_{\gamma_{0}}^{(1,1)}(\boldsymbol{r}=0)=-\boldsymbol{1} \int_{0}^{\infty} \mathrm{d} k k^{-1} J_{1 / 2}^{2}(k) \phi S_{\gamma_{0}}(k)\left[1+\phi S_{\gamma_{0}}(k)\right]^{-1}, \\
& \begin{aligned}
\boldsymbol{A}_{\gamma_{0}}^{(m, m+2)}(\boldsymbol{r}=0)= & \boldsymbol{A}_{\gamma_{0}}^{(m+2, m)}(\boldsymbol{r}=0)=-\frac{3}{2}(m+1) !(2 m-1) ! ! \Delta^{(m+1, m+1)} \\
& \times \int_{0}^{\infty} \mathrm{d} k k^{-1} J_{m-1 / 2}(k) J_{m+3 / 2}(k) \phi S_{\gamma_{0}}(k)\left[1+\phi S_{\gamma_{0}}(k)\right]^{-1},
\end{aligned} \\
& \boldsymbol{A}_{\gamma_{0}}^{(n, m)}(\boldsymbol{r}=0)=0, \quad \text { if } n \neq m \text { and } n \neq m \pm 2 .
\end{aligned}
$$

Here $(2 m-1) ! !=1 \cdot 3 \cdot 5 \cdot \ldots \cdot(2 m-3) \cdot(2 m-1)$; the $\boldsymbol{\Delta}$-tensors are defined in eq. (I-2.9). The equations (4.5)-(4.8) are the analoga of eqs. (I-6.11)-(I-6.14) for $\boldsymbol{A}_{n_{0}}^{(n, m)}(\boldsymbol{r}=0)$, and are obtained by performing the angular integration in eq. (4.3), using the explicit expressions for $\boldsymbol{A}^{(n, m)}(\boldsymbol{k})$.

Substitution of formula (4.5) into eq. (3.3) shows that $\gamma_{0}$ is indeed of the form (4.1) and gives for the scalar functions $\gamma_{0}^{(m)}$ the equations

$$
\begin{array}{r}
\gamma_{0}^{(m)}-\gamma_{0}^{(m)} \phi(2 m-1) \int_{0}^{\infty} \mathrm{d} k k^{-1} J_{m-1 / 2}^{2}(k) S_{\gamma_{0}}(k)\left[1+\phi S_{\gamma_{0}}(k)\right]^{-1}=n_{0} \\
m=2,3, \ldots
\end{array}
$$

One sees that $\gamma_{0}^{(m)}$ differs from $n_{0}$ by terms of order $\phi^{2}$.

In order to solve the infinite set of coupled equations (4.9) to a sufficient accuracy we approximate the function $S_{\gamma_{0}}(k)$ by

$$
S_{\gamma_{0}}^{(L)}(k) \equiv S(k)+\sum_{p=2}^{L} \frac{9}{2} \varepsilon_{p}\left(\gamma_{0}^{(p)}-n_{0}\right) n_{0}^{-1}(2 p-1)^{2} \frac{\pi}{2} k^{-3} J_{p-1 / 2}^{2}(k)
$$

for a given number $L=2,3, \ldots$ From the definition of $S(k)$ (eq. (I-6.6)) and 
$S_{\gamma_{0}}(k)$ (eq. (4.4)) it follows that

$$
\lim _{L \rightarrow \infty} S_{\gamma_{0}}^{(L)}(k)=S_{\gamma_{0}}(k)
$$

With the above approximation the $L-1$ equations for $\gamma_{0}^{(m)}(m=2,3, \ldots, L)$ in (4.9) decouple and may be solved numerically*. We give in table $I$, for volumefractions $\phi$ up to 0.45 , the values of $\phi \gamma_{0}^{(m)} / n_{0}(m=2,3,4,5)$ obtained by this procedure with $L=5$.

To calculate the diffusion coefficient $D(k)$ we shall in the next section use these values for $\gamma_{0}^{(m)}$; also, in expression (4.3) for $\boldsymbol{A}_{\gamma_{0}}^{(n, m)}(\boldsymbol{r})$, we shall approximate $S_{\gamma_{0}}(a k)$ by $S_{\gamma_{0}}^{(5)}(a k)$, as defined in eq. (4.10). An estimate of the error resulting from this approximation can be obtained by repeating the calculation of $\gamma_{0}$ described above to a lower order. In table II we give for $\phi=0.40$ the values of $\phi \gamma_{0}^{(m)} / n_{0}(2 \leqslant m \leqslant L)$ and $\boldsymbol{1}+\boldsymbol{A}_{\gamma_{0}}^{(1,1)}(\boldsymbol{r}=0)$, obtained from this calculation with $L$ ranging from 2 to 5 . One finds, in particular, that by increasing the order $L$ from 4 to 5 , the change in $\gamma_{0}^{(m)}(m=2,3,4)$ is smaller than $3 \%$, while the value of $\boldsymbol{1}+\boldsymbol{A}_{\gamma_{0}}^{(1,1)}(\boldsymbol{r}=0)$ changes by even less. This last quantity is equal to the large wavevector limit of $D(k)$, to lowest order in the expansion in correlationfunctions, cf. section 5. Moreover, it has been checked that also for smaller wavevectors use of $S_{\gamma_{0}}^{(4)}$ instead of $S_{\gamma_{0}}^{(5)}$ would change the (lowest order) results for $D(k)$ by not more than $2 \%$.

We thus conclude that the approximation made by replacing $S_{\gamma_{0}}$ by $S_{\gamma_{0}}^{(5)}$ (defined in eq. (4.10)) is for present purposes sufficiently accurate.

TABLE I

Values of the scalar functions $\phi \gamma_{0}^{(m)} / n_{0}(m=2,3,4$ and 5) for nine different volumefractions $\phi$

\begin{tabular}{lllll}
\hline \multicolumn{5}{c}{$\phi \gamma_{0}^{(m)} / n_{0}$} \\
\cline { 2 - 5 }$\phi$ & $m=2$ & $m=3$ & $m=4$ & $m=5$ \\
\hline 0.05 & 0.0553 & 0.0542 & 0.0533 & 0.0525 \\
0.10 & 0.1228 & 0.1177 & 0.1135 & 0.1104 \\
0.15 & 0.2048 & 0.1918 & 0.1813 & 0.1738 \\
0.20 & 0.3038 & 0.2777 & 0.2574 & 0.2432 \\
0.25 & 0.4224 & 0.3766 & 0.3423 & 0.3186 \\
0.30 & 0.5627 & 0.4895 & 0.4364 & 0.4005 \\
0.35 & 0.7267 & 0.6172 & 0.5402 & 0.4888 \\
0.40 & 0.9157 & 0.7601 & 0.6538 & 0.5839 \\
0.45 & 1.1310 & 0.9183 & 0.7776 & 0.6856 \\
\hline
\end{tabular}

* Use was made of numerical algorithms from the NAG library (Oxford). 
TABLE II

Values of $\phi \gamma_{0}^{(m)} / n_{0}(2 \leqslant m \leqslant L)$ for $\phi=0.40$, obtained by solving eq. (4.9) with the approximation of $S_{\gamma_{0}}$ by $S_{\gamma_{0}}^{(L)}$ (eq. (4.10)). The order $L$ of this approximation is increased from 2 to 5 , the value $L=5$ giving the results presented in the previous table for the whole range of volumefractions. Also shown, for $\phi=0.40$, is the convergence of the quantity $\mathbf{1}+\boldsymbol{A}_{\gamma_{0}}^{(1,1)}(\boldsymbol{r}=0)$, as the order of the approximation increases.

\begin{tabular}{|c|c|c|c|c|c|}
\hline \multirow[b]{2}{*}{$L$} & \multicolumn{4}{|c|}{$\phi \gamma_{0}^{(m)} / n_{0}(\phi=0.40)$} & \multirow[b]{2}{*}{$\mathbf{1}+\boldsymbol{A}_{\gamma_{0}}^{(1.1)}(r=0)$} \\
\hline & $m=2$ & $m=3$ & $m=4$ & $m=5$ & \\
\hline 2 & 0.783 & & & & 0.3971 \\
\hline 3 & 0.868 & 0.711 & & & 0.3631 \\
\hline 4 & 0.901 & 0.745 & 0.637 & & 0.3531 \\
\hline 5 & 0.916 & 0.760 & 0.654 & 0.584 & $0.348 \quad$ t \\
\hline
\end{tabular}

\section{Expansion of $D(k)$ in correlations of renormalized density fluctuations}

We now return to the formal expression (3.9) for the wavevector dependent diffusion coefficient. We first note that we may replace the two density operators $n$ in this expression by their fluctuations $\delta n=n-n_{0}$. The terms containing the average $n_{0}$ do not contribute, in view of the fact that

$$
\hat{k} \cdot \int \mathrm{d} \boldsymbol{r} \mathrm{e}^{-\mathrm{i} k \cdot r} \boldsymbol{A}_{\gamma_{0}}^{(\mathrm{1}, m)}\left(\boldsymbol{r}^{\prime}-\boldsymbol{r}\right)=\int \mathrm{d} \boldsymbol{r} \mathrm{e}^{\mathrm{i} k \cdot r} \boldsymbol{A}_{\gamma_{0}}^{(1, m)}\left(\boldsymbol{r}-\boldsymbol{r}^{\prime}\right) \cdot \hat{k}=0
$$

Indeed these integrals are proportional to either $\hat{k} \cdot \boldsymbol{A}^{(1, m)}(\boldsymbol{k})$ or to $\boldsymbol{A}^{(m, 1)}(\boldsymbol{k}) \cdot \hat{k}$ (cf. eq. (4.3)*), both of which quantities are zero for all $m$, as follows from eq. (I-2.15). The resulting exact expression for the diffusion coefficient $D(k)$

$$
G(k) D(k) / D_{0}=1+N^{-1}\left\langle\hat{k} \cdot\left\{P \delta n \mathscr{A}_{\gamma_{0}}\left(1-\delta \gamma Q \mathscr{B}^{-1} \mathscr{A}_{\gamma_{0}}\right)^{-1} \delta n P\right\}(k \mid k) \cdot \hat{k}\right\rangle
$$

is the starting point for an expansion of this quantity in correlationfunctions of the renormalized density fluctuations $\delta \gamma$ of higher and higher order.

To lowest order in $\delta \gamma$ one has

$$
G(k) D(k) / D_{0}=1+N^{-1}\left\langle\hat{k} \cdot\left\{\delta n \boldsymbol{A}_{\gamma_{0}}^{(1,1)} \delta n\right\}(\boldsymbol{k} \mid \boldsymbol{k}) \cdot \hat{k}\right\rangle .
$$

* Note that if the expression (4.3) for $\boldsymbol{A}_{\gamma_{0}}^{(n, m)}(\boldsymbol{r})$ is substituted into eq. (5.1), one may replace the connectorfield $\tilde{A}^{(n, m)}(r)$ in this expression by $\boldsymbol{A}^{(n, m)}(r)$, since these two connectorfields differ by a finite amount in a single point only (cf. eq. (2.15)). 
In $r$-representation the two-point correlation in this equation can be written as the sum of a self- and a pair-correlation (cf. eq. (3.10))

$$
\left\langle\left\{\delta n \boldsymbol{A}_{\gamma_{0}}^{(1,1)} \delta n\right\}\left(\boldsymbol{r} \mid \boldsymbol{r}^{\prime}\right)\right\rangle=n_{0} \boldsymbol{A}_{\gamma_{0}}^{(1,1)}(\boldsymbol{r}=0) \delta\left(\boldsymbol{r}^{\prime}-\boldsymbol{r}\right)+n_{0}^{2} \boldsymbol{A}_{\gamma_{0}}^{(1,1)}\left(\boldsymbol{r}^{\prime}-\boldsymbol{r}\right)\left[g\left(\left|\boldsymbol{r}^{\prime}-\boldsymbol{r}\right|\right)-1\right],
$$

where $g(r)$ is the pair distributionfunction. Transforming to wavevector representation according to eq. (2.9) one therefore finds for $D(k)$ to lowest order

$$
G(k) D(k) / D_{0}=1+\hat{k} \cdot \boldsymbol{A}_{\gamma_{0}}^{(1,1)}(\boldsymbol{r}=0) \cdot \hat{k}+n_{0} \int \mathrm{d} \boldsymbol{r} \mathrm{e}^{i k \cdot r} \hat{k} \cdot \boldsymbol{A}_{\gamma_{0}}^{(1,1)}(\boldsymbol{r}) \cdot \hat{k}[g(r)-1]
$$

To evaluate this expression we used (as in paper I, cf. appendix D) the Percus-Yevick approximation for the Fourier transform of the pair correlationfunction

$$
\nu(k) \equiv \int \mathrm{d} r \mathrm{e}^{\mathrm{i} k \cdot r}[g(r)-1]
$$

The structurefactor $G(k)$, defined as

$$
G(k)=1+n_{0} \nu(k)
$$

was calculated in the same approximation*.

The first two terms on the r.h.s. of eq. (5.5) are wavevector independent; from eq. (4.6) one finds

$$
1+\hat{k} \cdot \boldsymbol{A}_{\gamma_{0}}^{(1,1)}(\boldsymbol{r}=0) \cdot \hat{k}=\frac{2}{\pi} \int_{0}^{\infty} \mathrm{d} x(\sin x / x)^{2}\left[1+\phi S_{\gamma_{0}}(x)\right]^{-1}
$$

The function $S_{\gamma_{0}}(x)$ was discussed in the last section. The third term on the r.h.s. of eq. (5.5) is, according to eq. (4.3) $\dagger$, given by

$$
\begin{aligned}
& n_{0} \int \mathrm{d} \boldsymbol{r} \mathrm{e}^{\mathrm{i} k \cdot \boldsymbol{r}} \hat{k} \cdot \boldsymbol{A}_{\gamma_{0}}^{(1,1)}(\boldsymbol{r}) \cdot \hat{k}[g(r)-1]=n_{0}(2 \pi)^{-3} \int \mathrm{d} \boldsymbol{k}^{\prime} \hat{k} \cdot \boldsymbol{A}^{(1,1)}\left(\boldsymbol{k}^{\prime}\right) \cdot \hat{k} \\
& \quad \times\left[1+\phi S_{\gamma_{0}}\left(a k^{\prime}\right)\right]^{-1} \nu\left(\left|\boldsymbol{k}-\boldsymbol{k}^{\prime}\right|\right)
\end{aligned}
$$

* For the value of $G(k)$ at $k=0$, however, we used the slightly more accurate formula of Carnahan and Starling ${ }^{13}$ ).

$\dagger \mathrm{Cf}$. also the first footnote of this section. 
where (cf. eq. (I-2.15))

$$
n_{0} \boldsymbol{A}^{(1,1)}(k)=\frac{9}{2} \phi(a k)^{-4} \sin ^{2}(a k)(\boldsymbol{1}-\hat{k} \hat{k}) .
$$

The results from a numerical integration of these equations will be given in the next section. We note that for large wavevectors $k$ the integral (5.9) goes to zero and only the contribution (5.8) to the diffusion coefficient remains, which in this limit represents the selfdiffusion coefficient.

From eq. (5.2) one sees that the first correction to the result (5.3) for $D(k)$ is due to three-point correlations between renormalized density fluctuations. In general this correction will therefore contain the three-sphere correlationfunction and is difficult to evaluate. Nevertheless, an indication of the accuracy of our lowest order result for $D(k)$ can be obtained by calculating the self-diffusion coefficient $D_{\mathrm{s}}$ to higher order. Indeed $D_{\mathrm{s}}$ contributes to $D(k)$ at all wavevectors,

$$
G(k) D(k)=D_{\mathrm{s}}+k_{\mathrm{B}} T N^{-1} \sum_{i \neq j}\left\langle\hat{k} \cdot \boldsymbol{\mu}_{i j} \cdot \hat{k} \mathrm{e}^{i k \cdot \boldsymbol{R}_{y j}}\right\rangle
$$

(cf. eqs. (2.1) and (2.2)), and is in fact the largest of the two terms on the r.h.s. of eq. (5.11), over the whole range of wavevectors and densities. For this reason we shall in the remaining part of this section focus our attention on the selfdiffusion coefficient, given by eq. (3.11).

Upon expansion of expression (3.11) for $D_{\mathrm{s}}$ in correlations of renormalized density fluctuations, one finds for the zeroth order term $D_{s}^{(0)}$

$$
1 D_{\mathrm{s}}^{(0)} / D_{0}=1+\boldsymbol{A}_{\gamma_{0}}^{(1,1)}(r=0) .
$$

The r.h.s. of this equation is identical to eq. (5.8); the lowest order term therefore in the expansion of formula (3.11) for $D_{\mathrm{s}}$ is equal to the limit $k \rightarrow \infty$ of the lowest order term in the expansion of eq. (3.9) for $D(k)$. This correspondence, however, does not exist term by term for higher order terms. See in this connection the remark after eq. (3.11). The values of $D_{\mathrm{s}}^{(0)}$ (resulting from a numerical integration of the integral in eq. $\left.(5.8)^{*}\right)$ are shown in table III, for various volume-fractions up to $\phi=0.45$.

The lowest order correction $D_{\mathrm{s}}^{(2)}$ to $D_{\mathrm{s}}^{(0)}$ results from two-point correlations: it is given by (cf. eq. (3.11))

$$
1 D_{\mathrm{s}}^{(2)} / D_{0}=n_{0}^{-1} P\left\langle\left\{\mathscr{A}_{\gamma_{0}} \delta \gamma Q \mathscr{B}^{-1} \mathscr{A}_{\gamma_{0}} \delta n+\mathscr{A}_{\gamma_{0}} \delta \gamma Q \mathscr{B}^{-1} \mathscr{A}_{\gamma_{0}} \delta \gamma Q \mathscr{B}^{-1} \mathscr{A}_{\gamma_{0}} n_{0}\right\}(\boldsymbol{r} \mid \boldsymbol{r})\right\rangle P,
$$

* With the approximation of $S_{\gamma_{0}}$ by $S_{\gamma_{0}}^{(5)}$, cf. section 4 . 
TABLE III

Results from the evaluation of the fluctuation expansion of the selfdiffusion coefficient $D_{\mathrm{s}}$ to second order The contributions from the zeroth order term $D_{s}^{(0)}$ and the lowest order correction $D_{\mathrm{s}}^{(2)}$ thereto are specified separately

\begin{tabular}{ccc}
\hline$\phi$ & $D_{\mathrm{s}}^{(0)} / D_{0}+D_{\mathrm{s}}^{(2)} / D_{0}$ & $=D_{\mathrm{s}} / D_{0}$ \\
\hline 005 & $0887+0012$ & 090 \\
010 & $0781+0012$ & 079 \\
015 & $0685+0007$ & 069 \\
020 & $0598-0000$ & 060 \\
025 & $0521-0008$ & 051 \\
030 & $0454-0014$ & 044 \\
035 & $0397-0020$ & 038 \\
040 & $0348-0023$ & 033 \\
045 & $0307-0025$ & 028 \\
\hline
\end{tabular}

or, written out explicitly (cf. eqs. (3.5), (3.8), (3.10), (4.1) and (4.2))

$$
\begin{aligned}
& \boldsymbol{1} D_{\mathrm{s}}^{(2)} / D_{0}=\gamma_{0}^{(3)} n_{0}^{-1} \boldsymbol{A}_{\gamma_{0}}^{(13)}(\boldsymbol{r}=0) \odot \boldsymbol{B}^{(33)^{-1}} \odot \boldsymbol{A}_{\gamma_{0}}^{(3,1)}(\boldsymbol{r}=0) \\
& +2 \sum_{m=2}^{\infty} \gamma_{0}^{(m)} \gamma_{0}^{(m+2)} n_{0}^{-1} \int \mathrm{d} \boldsymbol{r} \boldsymbol{A}_{\gamma_{0}}^{(1 m)}(\boldsymbol{r}) \odot \boldsymbol{B}^{(m m)^{-1}} \odot \boldsymbol{A}_{\gamma_{0}}^{(m, m+2)}(\boldsymbol{r}=0) \\
& \odot \boldsymbol{B}^{(m+2 m+2)^{-1}} \odot \boldsymbol{A}_{\gamma_{0}}^{(m+21)}(-\boldsymbol{r}) \\
& +\sum_{m=2}^{\infty} \gamma_{0}^{(m)} \int \mathrm{d} \boldsymbol{r} \boldsymbol{A}_{\gamma_{0}}^{(1)}(\boldsymbol{r}) \odot \boldsymbol{B}^{(m, m)^{-1}} \odot \boldsymbol{A}_{\gamma_{0}}^{(m, 1)}(-\boldsymbol{r})[g(r)-1] \\
& +\sum_{m=2}^{\infty} \sum_{k=2}^{\infty} \gamma_{0}^{(m)} \gamma_{0}^{(k)} \int \mathrm{d} \boldsymbol{r} \int \mathrm{d} \boldsymbol{r}^{\prime} \boldsymbol{A}_{\gamma_{0}}^{(1, m)}(\boldsymbol{r}) \odot \boldsymbol{B}^{(m, m)^{-1}} \odot \boldsymbol{A}_{\gamma_{0}}^{(m, k)}\left(\boldsymbol{r}^{\prime}-\boldsymbol{r}\right) \\
& \odot \boldsymbol{B}^{(k, k)^{-1}} \odot \mathbf{A}_{\gamma_{0}}^{(k, 1)}\left(-\boldsymbol{r}^{\prime}\right)\left[g\left(\left|\boldsymbol{r}^{\prime}-\boldsymbol{r}\right|\right)-1\right] .
\end{aligned}
$$

To simplify this expression we have also used eqs. (4.7) and (4.8). The above equations (5.12)-(5.14) are the analoga of eqs. (I-5.7), (I-5.9) and (I-7.3), which give the first two terms of the expansion of $D_{\mathrm{s}}$ in correlations of unrenormalızed density fluctuations. Note however that the present expression for $D_{s}^{(2)}$ does not contain terms with factors $\boldsymbol{A}_{\gamma_{0}}^{(m, m)}(\boldsymbol{r}=0)$, since these diagonal ring-selfcorrelations are here already accounted for in the zeroth order term $D_{s}^{(0)}$, cf. the discussion in section 3 . This is in contrast to the expansion given in paper I, where corresponding factors did occur in the second order term (eq. $(\mathrm{I}-7.3))$. 
The above lowest order correction $D_{s}^{(2)}$ may be evaluated using the results of section 4 (cf. the similar calculation in paper $\mathrm{I}$, appendix D). As in paper I, we have restricted ourselves to a numerical evaluation of those terms in eq. (5.14) which do not contain connectors $\boldsymbol{A}_{\gamma_{0}}^{(n, m)}$ with $n$ or $m$ larger than 2 . This amounts to a restriction to corrections from monopole-dipole and dipole-dipole hydrodynamic interactions between density fluctuations. The results can be found in table III.

\section{Results and discussion}

In the previous sections we have calculated the concentration dependence of the wavevector dependent (short-time) diffusion coefficient $D(k)$ for spherical particles in suspension. For this purpose we derived the exact expression (5.2), from which one can obtain $D(k)$ as an expansion in correlationfunctions of higher and higher order. The lowest order term in this expansion (eq. (5.5)) fully contains the many-body hydrodynamic interactions between an arbitrary number of spheres. Moreover, the contributions from a special class of correlations, the so-called (diagonal) ring-selfcorrelations, are included in this term.

For the particular case of the (short-time) self-diffusion coefficient $D_{\mathrm{s}}$ (which is the large wavevector limit of $D(k)$ and is given by eq. (3.11)) we were able to calculate not only the zeroth order term $D_{s}^{(0)}$ (eq. (5.12)), but also the lowest order correction $D_{\mathrm{s}}^{(2)}$ thereto (eq. (5.14)), which is due to two-point correlations. In fig. 1 we have plotted $D_{\mathrm{s}}^{(0)} / D_{0}$ and $\left(D_{\mathrm{s}}^{(0)}+D_{\mathrm{s}}^{(2)}\right) / D_{0}$ as a function of the

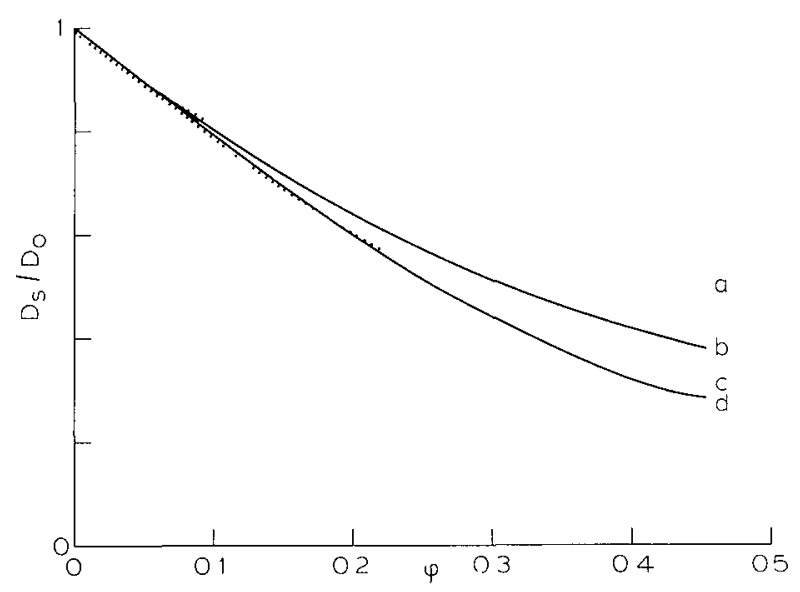

Fig 1. Results for the selfdiffusion coefficient $D_{\mathrm{s}}$ from the first two terms of the expansion in correlationfunctions considered here (curve c corresponding to $D_{\mathrm{s}}^{(0)}$, curve d to $D_{\mathrm{s}}^{(0)}+D_{\mathrm{s}}^{(2)}$ ) and from the expansion of paper I (curve a corresponding to $D_{\mathrm{s}}^{(0)}(\mathrm{I})$, curve b to $D_{\mathrm{s}}^{(0)}(\mathrm{I})+D_{\mathrm{s}}^{(2)}(\mathrm{I})$ ) 
volumefraction $\phi$ (from table III). In the same figure we have also shown the corresponding results from the alternative expansion of $D_{\mathrm{s}}$ considered in paper I: there the zeroth order term $D_{s}^{(0)}(\mathrm{I})$ contained no contributions due to correlations. If one compares the zeroth order results $D_{s}^{(0)}$ and $D_{s}^{(0)}(\mathrm{I})$ from these two alternative expansions (the two dotted curves in fig. 1), one sees that due to the inclusion of contributions from ring-selfcorrelations the values for $D_{\mathrm{s}}$ in the absence of correlations decrease by almost $40 \%$ at the highest volumefractions. Moreover, the lowest order correction $D_{s}^{(2)}$ is in the present expansion at most $8 \%$ of $D_{\mathrm{s}}^{(0)}$, whereas the corresponding term $D_{\mathrm{s}}^{(2)}(\mathrm{I})$ in the expansion considered in paper I was $20 \%$ of $D_{s}^{(0)}(I)$, at the highest volumefractions.

We conclude therefore, that the present expansion-resulting from an (algebraic) resummation of a special class of correlations-provides a more reliable zeroth order result for the diffusion coefficient than the expansion of paper I. We note that to linear order in the density these two expansions are, however, identical*.

As argued in section 5, one may use an error estimate for $D_{\mathrm{s}}$ to obtain an indication of the accuracy of our lowest order result for $D(k)$. Indeed $D_{\mathrm{s}} / G(k)$ (where $G(k)$ is the structurefactor) gives at all wavevectors the largest contribution to $D(k)$, which may also be written as (cf. eq. (5.11))

$$
G(k) D(k)=D_{\mathrm{s}}+k_{\mathrm{B}} T N^{-1} \sum_{i \neq j}\left\langle\hat{k} \cdot \boldsymbol{\mu}_{i j} \cdot \hat{k} \mathrm{e}^{\mathrm{i} \boldsymbol{k} \cdot \boldsymbol{R}_{i i}}\right\rangle .
$$

To lowest order the r.h.s. of the above equation is given by eq. (5.5) and contains $D_{\mathrm{s}}^{(0)}$ (cf. eq. (5.12)). It is found that adding the correction $D_{s}^{(2)}$ to $D_{\mathrm{s}}^{(0)}$ changes this lowest order result for $D(k)$ by less than $10 \%$ for wavevectors $a k \geqslant 3$ (where $a$ is the radius of the suspended spheres). This remains the case for all values of the wavevector if the volumefraction $\phi$ does not exceed 0.3 . However, at small wavevectors and the highest densities considered, our lowest order results for $D(k)$ become increasingly less accurate due to a near cancellation of the two terms on the r.h.s. of eq. (6.1).

In figs. 2 and 3 we have plotted for five values of the volumefraction $\phi$ the results $\dagger$ for $D(k) G(k) / D_{0}$ (which is the longitudinal part of the wavevector dependent sedimentation velocity, relative to its value at infinite dilution) and for $D_{0} / D(k)$. Note that in the absence of hydrodynamic interactions the first quantity is identically 1 and the second quantity equals the structurefactor

\footnotetext{
* This results from the fact-observed in section 4 - that the renormalized density differs from the real density by terms of order $\phi^{2}$.

$\dagger$ The values plotted contain the lowest order values calculated from eq. (5.5) to which the correction $D_{\mathrm{s}}^{(2)}$ (given in table III) has been added. In this way the values for $D_{\mathrm{s}}$ given in fig. 4 are obtained from figs. 2 and 3 in the limit $k \rightarrow \infty$.
} 


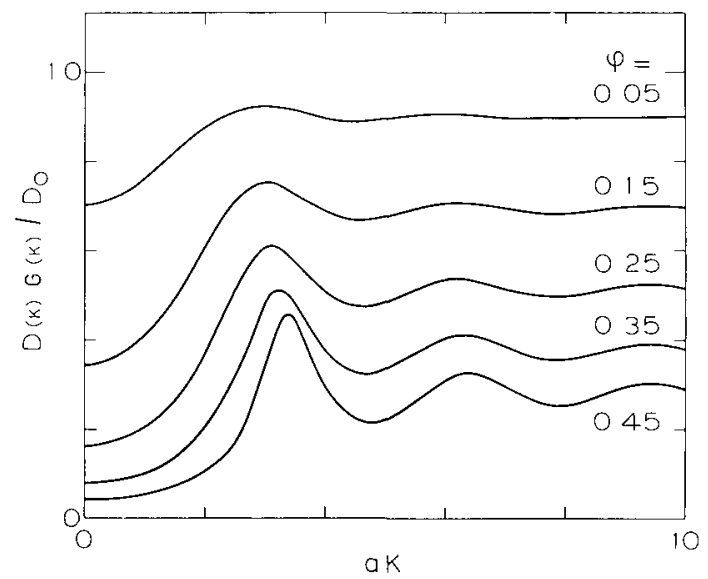

Fig 2 Wavevector dependence of $D(k) G(k) / D_{0}$ for five values of the volumefraction $\phi$

$G(k)$. A comparison with experiments is possible for the large and small wavevector limits of $D(k)$,

$$
D_{\mathrm{s}}=\lim _{k \rightarrow \infty} D(k), \quad D_{\mathrm{c}}=\lim _{k \rightarrow 0} D(k),
$$

which are the (short-time) self- and collective diffusion coefficients respectively. In fig. 4 we have plotted the theoretical values for these two coefficients, together with experimental results, ${ }^{411,12}$ ).

The diffusion coefficient at small wavevectors has been measured, by means of dynamic light-scattering, by Cebula, Ottewill, Ralston and Pusey ${ }^{11}$ ) for

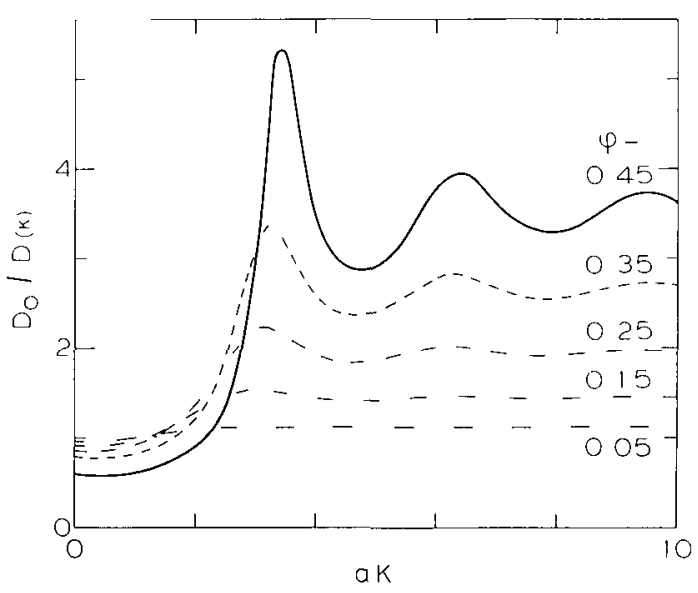

Fig 3 Wavevector dependence of $D_{0} / D(k)$ for five values of the volumefraction $\phi$ 
microemulsion droplets and by Kops-Werkhoven and Fijnaut ${ }^{12}$ ) for silica particles. These experiments both indicate that the collective diffusion coefficient is rather insensitive to changes in the concentration over a large range of volumefractions. This remarkable result is confirmed by our calculations of $D_{c}$, shown in fig. 4 for volumefractions $\phi \leqslant 0.3$ (as we remarked above, at higher concentrations our small wavevector results become less and less reliable due to cancellations). One should keep in mind, however, that on the timescale* of these experiments ${ }^{11,12}$ ) a particle diffuses over a distance of several radii, whereas our results are-strictly speaking - valid only for short times in which the configuration of the particles remains essentially constant.

Pusey and van Megen ${ }^{4}$ ) measured the diffusion coefficient of latex particles of radius $a=600 \mathrm{~nm}$, at large wavevectors $k \approx 18 / a$ for which $D(k)$ has attained its large- $k$ limit. The timescale of these measurements is such that a particle diffuses over a distance of about $a / 10$. For the densities considered one may therefore assume that the configuration of the particles is essentially constant on this timescale and that the measured quantity is indeed, as argued by Pusey and van Megen, the short-time selfdiffusion coefficient. One sees from fig. 4 that the theoretical results for $D_{\mathrm{s}}$ agree with the measurements up to the highest volumefractions. We recall that in paper I good agreement was obtained only for $\phi \leqslant 0.3$.

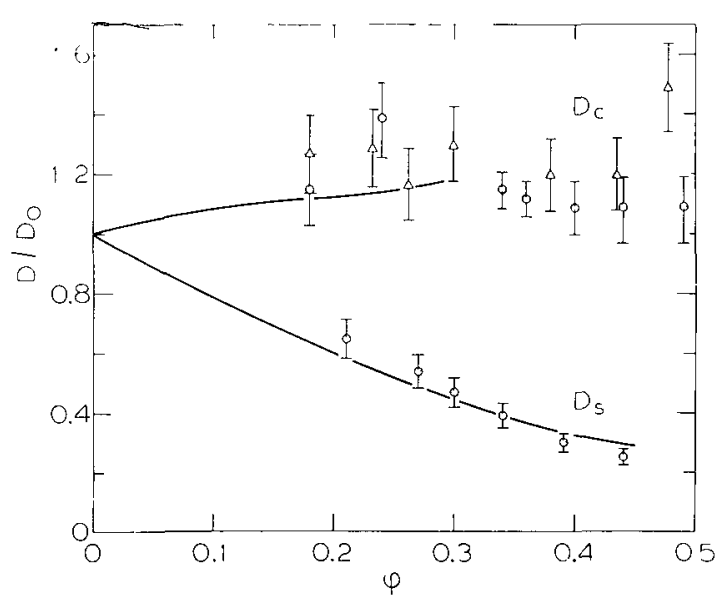

Fig. 4. Density dependence of the (short-time) self- and collective diffusion coefficients, $D_{\mathrm{s}} / D_{0}$ and $D_{\mathrm{c}} / D_{0}$ respectively. The solid curves correspond to the values given in fig. 3 , in the two limits of large and small wavevectors. Experimental data for $D_{\mathrm{s}}$ are taken from ref. 4 (lower dots); the data for $D_{\mathrm{c}}$ are taken from refs. 11 (triangles) and 12 (upper dots).

\footnotetext{
* This timescale is the decay-time of the electric field autocorrelation function, which is of the order of $\left(D_{0} k^{2}\right)^{-1}$.
} 


\section{Interpretation in terms of an effective pair-mobility}

Our lowest order result (5.5) for the diffusion coefficient can be written in a form similar to eq. (2.1)

$$
D(k)=k_{\mathrm{B}} T[N G(k)]^{-1} \sum_{i, j=1}^{N}\left\langle\hat{k} \cdot \boldsymbol{\mu}_{l j}^{\mathrm{eff}} \cdot \hat{k} \mathrm{e}^{\mathrm{ik} \cdot \boldsymbol{R}_{\eta}}\right\rangle,
$$

with $\mu_{1 j}^{\text {eff }}$ given by (cf. eqs. (5.8)-(5.10))

$$
\begin{aligned}
\boldsymbol{\mu}_{l y}^{\text {eff }}= & (6 \pi \eta a)^{-1} \frac{9}{2}\left(\frac{4}{3} \pi a^{3}\right)(2 \pi)^{-3} \int \mathrm{d} \boldsymbol{k} \mathrm{e}^{-1 k \cdot R_{y}}(\boldsymbol{1}-\hat{k} \hat{k})(a k)^{-4} \sin ^{2}(a k) \\
& \times\left[1+\phi S_{\gamma_{0}}(a k)\right]^{-1} .
\end{aligned}
$$

This quantity depends only on $\boldsymbol{R}_{l}$ and $\boldsymbol{R}$, and may therefore be interpreted as an effective pair-mobility. The renormalization factor $\left[1+\phi S_{\gamma_{0}}(a k)\right]^{-1}$ in this expression accounts for the many-body hydrodynamic interactions between an arbitrary number of spheres, including contributions from (diagonal) ringselfcorrelations.

For small values of $a k, S_{\gamma_{0}}(a k)$ behaves as

$$
S_{\gamma_{0}}(a k)=\frac{5}{2} \gamma_{0}^{(2)} / n_{0}+\mathscr{O}(a k)^{2},
$$

as follows from expansion of definition (4.4). Since the largest contribution to the integral in eq. (7.2) arises from small values of $a k$, one may approximate $S_{\gamma_{0}}(a k)$ in the integrand by its small- $k$ limit (the numerical consequences of this approximation for $D(k)$ are discussed below). One then has for the effective pair-mobility the simple expression (cf. the evaluation of the connector $\boldsymbol{A}_{i v}^{(1,1)}$ in ref. 8)

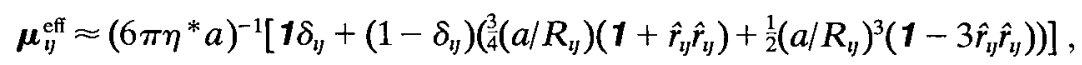

with the definition

$$
\eta^{*} \equiv \eta\left(1+\frac{5}{2} \phi \gamma_{0}^{(2)} / n_{0}\right) .
$$

The vector $\boldsymbol{R}_{l j} \equiv \boldsymbol{R}_{j}-\boldsymbol{R}_{\imath}$ has magnitude $\boldsymbol{R}_{l j}$ and direction $\hat{r}_{i j} \equiv \boldsymbol{R}_{\imath j} / R_{l j}$. The renormalized density $\gamma_{0}^{(2)}$ is given as a function of $n_{0}$ in table I.

If one calculates $D(k)$ from eq. (7.1), with the approximation (7.4) (using the Percus-Yevick pair correlationfunction), one finds values for $D(k)$ which are smaller than the results* shown in fig. 3, especially at small wavevectors. For

${ }^{*}$ We recall that these values result from eqs. (7.1) and (7.2), with the addition of the correction $D_{s}^{(2)} / G(k)$, from table III. 
$a k \geqslant 3$, however, the difference is less than $10 \%$, over the whole range of volumefractions For selfdiffusion in particular, one finds from eq (74) that (cf eq (2 2))

$$
D_{\mathrm{s}} \approx k_{\mathrm{B}} T(6 \pi \eta * a)^{-1}
$$

This formula differs from our full result (fig 4 ) by at most $7 \%$

The expression (74) for the effective pair-mobility has a simple physical interpretation it is the mobility tensor - up to terms of order $\left(a / R_{i j}\right)^{4}$ - of two spheres, in a fluid with viscosity $\eta^{*}$ It can be shown ${ }^{14}$ ) that, within the order of approximation of eq $(71), \eta^{*}$ equals the effective viscosity of the suspension To linear order in the density this identification is in fact exact*, since $\gamma_{0}^{(2)}=n_{0}+\mathscr{O}\left(\phi^{2}\right)$ (cf remark after eq (49)), so that

$$
\eta^{*}=\eta\left(1+{ }_{2}^{5} \phi+\mathscr{O}\left(\phi^{2}\right)\right),
$$

which is Einstein's result for the effective viscosity

We stress the fact (noted also in paper I) that the hydrodynamic interaction between two particles in a suspension is not screened by the presence of the other particles By this we mean that the effective pair-mobility discussed above is of long range (1t falls off as $1 / R$ ) In contrast, Snook, van Megen and Tough ${ }^{15}$ ) recently proposed an empirical screened pair-mobility to reproduce the experimental data for the diffusion coefficient In view of the above, there does not appear to be a physical motivation for their choice

To avold misunderstanding, it should be mentioned that screening of hydrodynamic interactions does occur in a different system, viz in a porous medium consisting of immobile particles in a viscous fluid (see e g ref 16) The properties of such a medium - which are different from those of a suspension, in which the particles may move freely-were studied (in particular for large concentrations of the particles) by Muthukumar ${ }^{17}$ ), including also the effect of many-body hydrodynamic interactions

\section{Acknowledgement}

This work was performed as part of the research programme of the "Stıchting voor Fundamenteel Onderzoek der Materie" (FOM), with financial support from the "Nederlandse Organisatie voor Zuiver Wetenschappelijh Onderzoek" ( $\mathrm{Z}$ W O )

* This was essentially observed already in paper I eq (82) 


\section{Appendix}

Proof of eq. (3.7)

We start from the identity

$$
\mathscr{A}\left(1-n Q \mathscr{B}^{-1} \mathscr{A}\right)^{-1} n=\mathscr{A}_{\gamma_{0}}\left[1-\left(n-\gamma_{0}\right) Q \mathscr{B}^{-1} \mathscr{A}_{\gamma_{0}}\right]^{-1} n
$$

where $\mathscr{A}_{\gamma_{0}}$ has been defined in eq. (3.2). It is convenient to define an operator $I$ with kernel

$$
I\left(\boldsymbol{r} \mid \boldsymbol{r}^{\prime}\right)= \begin{cases}1, & \text { if } \boldsymbol{r}=\boldsymbol{r}^{\prime} \\ 0, & \text { if } \boldsymbol{r} \neq \boldsymbol{r}^{\prime}\end{cases}
$$

and a matrix $\mathscr{B}_{\gamma_{0}}$ with elements

$$
\left\{\mathscr{B}_{\gamma_{0}}\right\}_{n, m}=\delta_{n m} \boldsymbol{A}_{\gamma_{0}}^{(m, m)}(\boldsymbol{r}=0)
$$

With these notations we can write

$$
\mathscr{A}_{\gamma_{0}}=\mathscr{\mathscr { A }}_{\gamma_{0}}+\mathscr{B}_{\gamma_{0}} I
$$

where $\mathscr{A}_{\gamma_{0}}$ is defined in eq. (3.8). In the same compact notation we have for $\gamma \equiv \gamma_{0} n_{0}^{-1} n$,

$$
\gamma=n\left(1-\mathscr{Q B}^{-1} \mathscr{B}_{\gamma_{0}}\right)^{-1}
$$

cf. eqs. (3.1) and (3.3).

We note that as a consequence of the fact that $\mathscr{A}_{\gamma_{0}} I=0$, one has the identity

$$
\mathscr{A}_{\gamma_{0}}=\mathscr{A}_{\gamma_{0}}\left(1-\gamma_{0} Q_{\mathscr{B}^{-1}} \mathscr{B}_{\gamma_{0}} I\right)^{-1}
$$

Upon substitution into the r.h.s. of eq. (A.1) and repeated use of definition (A.4) one then finds

$$
\begin{aligned}
& \mathscr{A}\left(1-n Q \mathscr{B}^{-1} \mathscr{A}\right)^{-1} n=\mathscr{A}_{\gamma_{0}}\left(1-n Q \mathscr{B}^{-1} \mathscr{A}_{\gamma_{0}}+\gamma_{0} Q \mathscr{B}^{-1} \mathscr{A}_{\gamma_{0}}\right)^{-1} n \\
& \quad=\mathscr{A}_{\gamma_{0}}\left(1-\left(1-n Q \mathscr{B}^{-1} \mathscr{B}_{\gamma_{0}} I\right)^{-1}\left(n-\gamma_{0}\right) Q \mathscr{B}^{-1} \mathscr{A}_{\gamma_{0}}^{\circ}\right)^{-1}\left(1-n Q \mathscr{B}^{-1} \mathscr{B}_{\gamma_{0}} I\right)^{-1} n
\end{aligned}
$$

We now use the identity

$$
\left(1-n Q \mathscr{B}^{-1} \mathscr{B}_{\gamma_{0}} I\right)^{-1} \gamma_{0} Q \mathscr{B}^{-1} \mathscr{\mathscr { A }}_{\gamma_{0}}=\gamma_{0} Q \mathscr{B}^{-1} \mathscr{\mathscr { A }}_{\gamma_{0}}
$$


which follows from $I \mathscr{A}_{\gamma_{0}}=0$, and another identity

$$
\left(1-n Q \mathscr{B}^{-1} \mathscr{B}_{\gamma_{0}} I\right)^{-1} n=n\left(1-Q \mathscr{B}{ }^{1} \mathscr{B}_{\gamma_{0}}\right)^{-1} \equiv \gamma
$$

(cf eq (A 5)) Eq (A 9) is a consequence of the fact that $n I n=n$

Substituting eqs (A 8) and (A 9) into eq (A 7), one then finds

$$
\mathscr{A}\left(1-n Q \mathscr{B}^{-1} \mathscr{A}\right)^{-1} n=\mathscr{A}_{\gamma_{0}}\left(1-\delta \gamma Q \mathscr{B}^{-1} \mathscr{A}_{\gamma_{0}}\right)^{-1} \gamma
$$

where $\delta \gamma \equiv \gamma-\gamma_{0}$ This is the required formula (37)

\section{References}

1) C W J Beenakker and P Mazur, Physıca 120A (1983) 388

2) P N Pusey and R J A Tough, J Phys A15 (1982) 1291

3) P N Pusey and R J A Tough, in Dynamic Light-scattering and Velocimetry, R Pecora, ed (Plenum, New York, to be published)

4) P N Pusey and W van Megen, J de Phys 44 (1983) 285

5) W B Russel and A B Glendinning, J Chem Phys 74 (1981) 948

6) HM Fijnaut, J Chem Phys 74 (1981) 6857

7) C W J Beenakker and P Mazur, Phys Lett 91A (1982) 290

8) P Mazur and W van Saarloos, Physica 115A (1982) 21

9) $\mathrm{T}$ Yoshızakı and H Yamakawa, J Chem Phys 73 (1980) 578

10) C W J Beenakker and P Mazur, Phys Lett 98A (1983) 22

11) D J Cebula, RH Ottewill, J Ralston and PN Pusey, J Chem Soc Faraday Trans 1 77 (1981) 2585

12) M M Kops-Werkhoven and H M Fijnaut, J Chem Phys 77 (1982) 2242

13) N F Carnahan and K E Starlıng, J Chem Phys 51 (1969) 635

14) C W J Beenakker, Physica A (1984) to be published

15) I Snook, W van Megen and R J A Tough, J Chem Phys 78 (1983) 5825

16) G K Batchelor, Ann Rev of Fluid Mech 6 (1974) 227

17) M Muthukumar, J Chem Phys 77 (1982) 959 